\title{
SmartPIV - an app for flow visualization by cross-correlation and optical flow using smartphones
}

\author{
Patrick Mäder ${ }^{1 *}$, Constanze Poll ${ }^{1}$, Jonas Hüther ${ }^{1}$, Sebastian Jeschke ${ }^{1}$, Henning \\ Otto $^{2}$, Christian Cierpka ${ }^{2 * *}$
}

${ }^{1}$ Technische Universität Ilmenau, Data-intensive Systems and Visualization Group, Ilmenau, Germany

${ }^{2}$ Technische Universität Ilmenau, Institute of Thermodynamics and Fluid Mechanics, Ilmenau, Germany

*patrick.maeder@tu-ilmenau.de, ${ }^{* *}$ christian.cierpka@tu-ilmenau.de

In recent years smartphones considerably changed our communication and are used on a daily (or even every minute) basis especially by students without any difficulties. Fluid flows also belong to our daily experiences. However, the education of the basic principles of fluid mechanics is sometimes cumbersome due to its non-linear nature. This problem may be tackled in practical sessions applying flow visualization techniques in wind or water tunnels and directly learn from own observations. Nowadays, often optical methods like particle imaging velocimetry (PIV) or particle tracking velocimetry (PTV) are used for these purposes. A typical PIV/PTV setup consists of a (double)pulse laser, a scientific camera and a synchronization device. The costs for this equipment can easily add up to more than 100,000 euros and the installations and set up of the systems requires experiences and is complex. For these reasons Universities often only offer practical courses for a small amount of students and the students may not be allowed to use and set up the systems by their own as the equipment is also needed for scientific research. Due to the COVID-19 pandemic it is also often not allowed to share equipment or even to work in larger groups during practical sessions.

However, modern smartphones offer a great selection of different sensors and are easy to set up, at least for students. The high frame rates of several hundred $\mathrm{Hz}$ provided by modern smartphones also enable their use for PIV. Cierpka et al. (2016) have shown that it is possible to use a smartphone with a cw-laser for reliable velocity estimates in a plane. Käufer et al. (2021) extended the system to stereoscopic PIV using two action cameras and a modulated cw-laser and Aguirre-Pablo et al. (2017) even used smartphones with colored LEDs for a tomographic reconstruction of the velocity field in a water jet. All these attempts were based on recorded videos that were later processed on a computer with conventional PIV software. However, only recently a survey among engineering students showed, that there is a strong interest in a mobile application (app) to perform PIV measurements (Minichiello et al., 2021). Therefore, the aim of the current study was to provide an app that allows for a direct (on-line) evaluation of the data in order to enable the students to directly see how the flow behavior changes when certain boundary conditions are varied and to already estimate if the data processing with the current video/evaluation setting will be successful. The app can also be used to determine the different effects of parameter changes for the PIV evaluation later at home.

The app was developed within a student software project in close collaboration between students of engineering and computer science. It is available for Android and iOS in the app stores and can be downloaded and used free of charge (TU Ilmenau, 2021). Since the ability for on-line flow visualization was most crucial concerning computer performance also optical flow algorithms were implemented as these request much less resources. After choosing the processing parameters (cp. Fig. 11, right) the user can directly process the images from the live preview (cp. Fig. 1, left insert), record videos or process already stored videos.

Images as well as videos can be stored for later export for lab reports. In addition to the images a text file and a csv file containing the data and parameter settings will be created that can be used for later analysis for example to test different mean estimators and outlier detection methods that will typically be taught in lab courses. Since for the conversion of the particle image displacement to physical coordinates the knowledge of the optical magnification is necessary a module that allows for a quick calibration using a square on a white paper with known side length was also included. Since typical fields of view are in the range of 1$20 \mathrm{~cm}$ the side length of the calibration target can be adopted to these values. The app can be intuitively controlled by users that are familiar with the video or photo function of their smartphones as the menus are designed very similar. A photograph of the application of SmartPIV for a test flow of a cylinder wake using a low power cw-laser diode is shown in Fig. 11(left). 

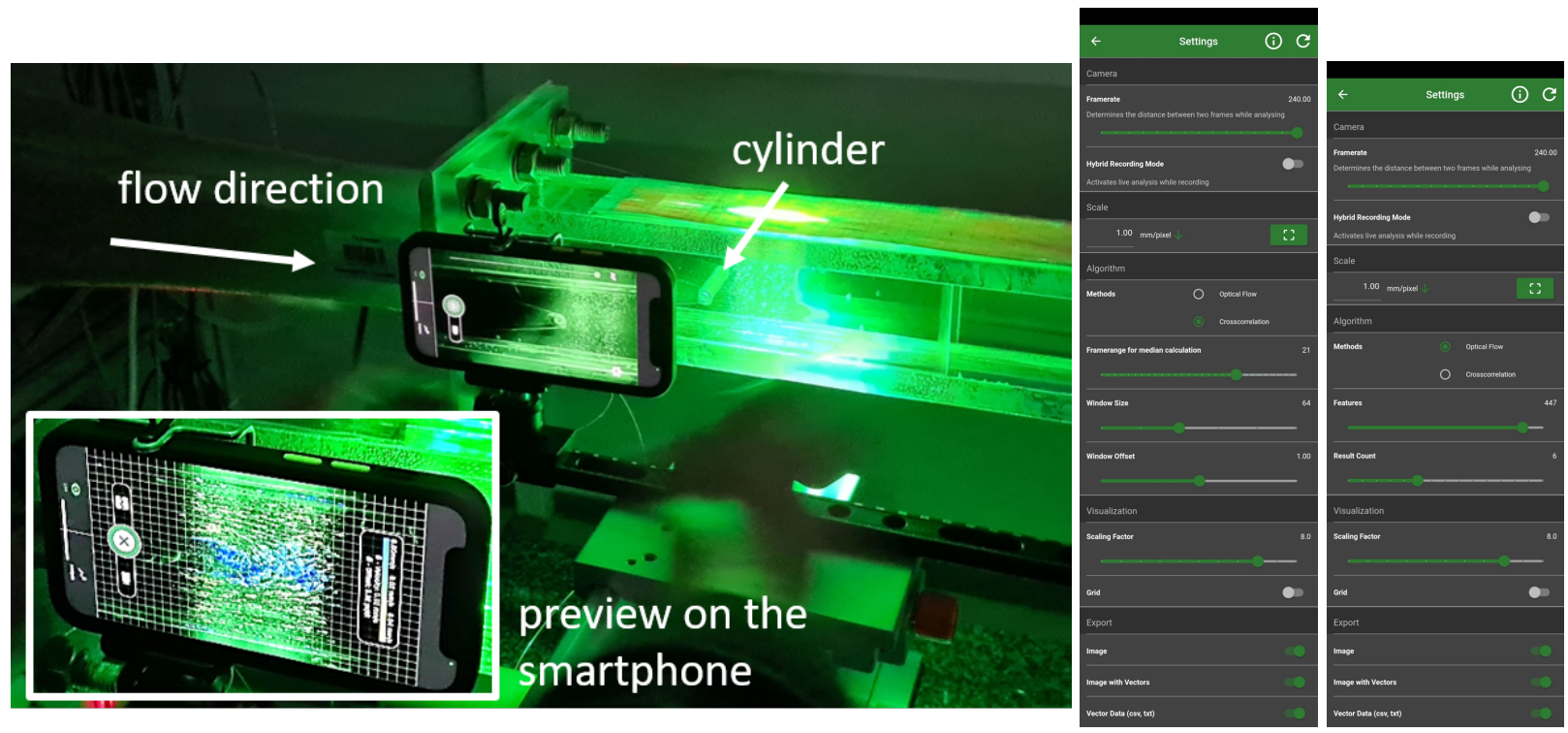

Figure 1: Experimental setup for a students exercise evaluation the flow past a cylinder. The setup includes a cw-laser diode for illumination and the smartphone with the preview screen of the SmartPIV app (left). Menu for the different evaluation methods optical flow or cross-correlation (right).

In the presentation the implementation on the smartphones and the accuracy will be discussed and a typical lab setup will be demonstrated.

\section{Acknowledgements}

Financial support in the frame of the fellowships for "Innovations for digital teaching" from the Thüringer Ministerium für Wirtschaft, Wissenschaft und Digitale Gesellschaft are gratefully acknowledged. The authors would also like to thank all the students, namely Julia Bruischütz, Teresa Bravo Roger, Jonas Stephan, Jonas Hiese, Marcus Orban, Marcel John, Christian Engelhardt as well as Jörg König for the support in the lab.

\section{References}

Aguirre-Pablo AA, Alarfaj MK, Li EQ, Hernández-Sánchez JF, and Thoroddsen ST (2017) Tomographic particle image velocimetry using smartphones and colored shadows. Scientific Reports 7:3714

Cierpka C, Hain R, and Buchmann NA (2016) Flow visualization by mobile phone cameras. Experiments in Fluids 57:108

Käufer T, König J, and Cierpka C (2021) Stereoscopic PIV measurements using low-cost action cameras. Experiments in Fluids 62:57

Minichiello A, Armijo D, Mukherjee S, Caldwell L, Kulyukin V, Truscott T, Elliott J, and Bhouraskar A (2021) Developing a mobile application-based particle image velocimetry tool for enhanced teaching and learning in fluid mechanics: A design-based research approach. Computer Applications in Engineering Education 29:517-537

TU Ilmenau (2021) SmartPIV.https://play.google.com/store/apps/details?id=de.tu_ilmenau. secsy.smartpiv/https://apps.apple.com/us/app/smartpiv/id1471308387 